\title{
The Shortwave (SW) Clear-Sky Detection and Fitting Algorithm: Algorithm Operational Details and Explanations
}

Revision 1, January 2004

C. N. Long and K. L. Gaustad

Pacific Northwest National Laboratory

Richland, Washington

Work supported by the U.S. Department of Energy,

Office of Energy Research, Office of Health and Environmental Research 


\section{Contents}

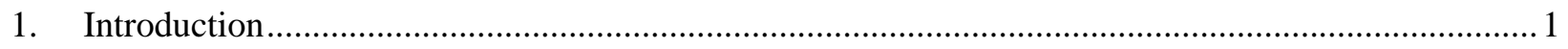

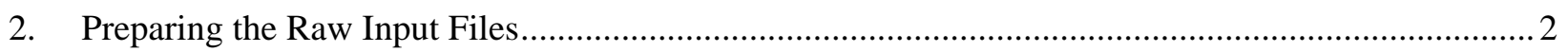

3. Running the Clear ID and Fitting Algorithm (swclrid1a) ........................................................... 4

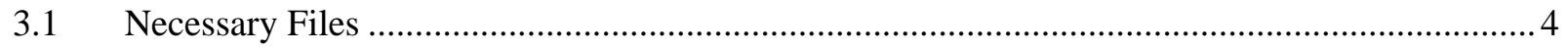

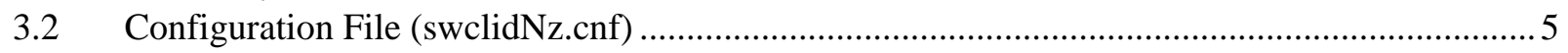

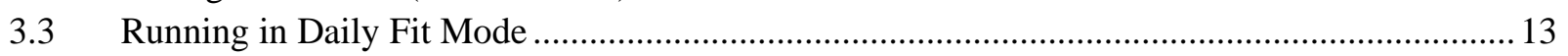

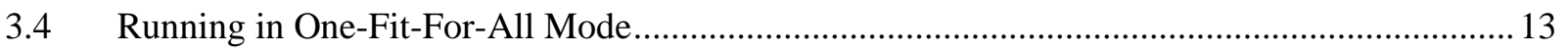

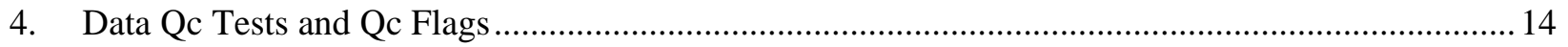

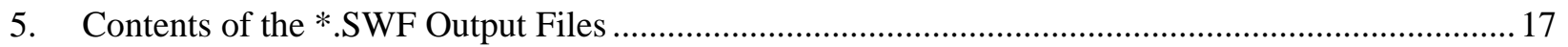

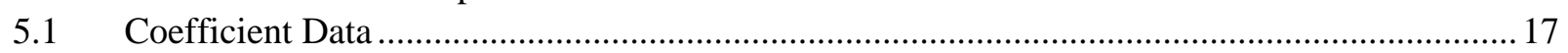

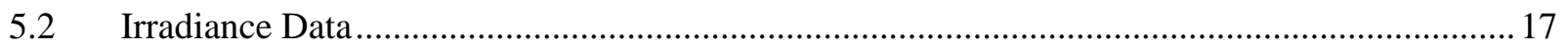

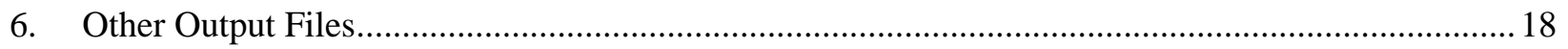

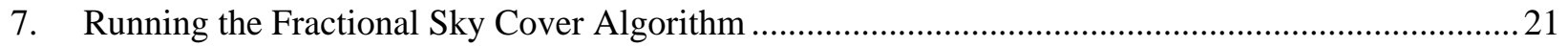

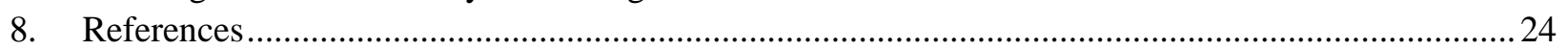




\section{Introduction}

This document describes some specifics of the algorithm for detecting clear skies and fitting clear-sky shortwave (SW) functions described in Long and Ackerman (2000). This algorithm forms the basis of the ARM SW FLUX ANAL 1Long VAP. In the Atmospheric Radiation Measurement (ARM) case, the value added procedures (VAP) can be described as having three parts: a "front end," a "black box," and a "back end." The "front end" handles the file management of the processing, what range of data files to process in the run, which configuration file to use for each site, extracting the data from the ARM NetCDF files into an ASCII format for the code to process, etc. The "back end" produces ARM-format NetCDF files of the output and other file management. The "black box" is the processing code(s), and is what is discussed in this document. Details on the "front" and "back" ends of the ARM VAP are presented elsewhere.

Before proceeding, it is highly recommended that the reader first review the documents:

Long, C. N. and T. P. Ackerman, 2000: "Identification of clear skies from broadband pyranometer measurements and calculation of downwelling shortwave cloud effects.” JGR, 105, No. D12, 15609-15626.

Long, C. N., T. P. Ackerman, J. J. DeLuisi, and J. Augustine, 1999: Estimation of fractional sky cover from broadband SW radiometer measurements.” Proc. 10th Conf. on Atmos. Rad., June 28-July 2, 1999, Madison, Wisconsin.

These Documents, available at http://science.arm.gov/ clong , "Clear Sky ID and Cloud Effect Paper" and "SW Sky Cover Retrieval Paper", detail the science and methodology behind the algorithm; subjects not covered in this document and without which some of the following discussion might not be readily understandable. The intent of this document is to provide details sufficient to run the code(s) and understand some parts included in the code(s) (such as the data QC checks) not covered in the above papers, as well as detail the code outputs and some suggested uses for the output data. These codes are written in FORTRAN77, and are available from the author as stand-alone programs. The wording that follows is derived in part from documentation provided along with the PC Win95/98/NT version of the code currently in use by the atmospheric science community, such as members of the Baseline Surface Radiation Network, under the name "swclidNz.zip." Thus some references are tailored to the personal computer (PC) environment, such as the referrals to batch files. For the UNIX environment, specifics will need to be garnered from the ARM SW FLUX ANAL 1Long "front" and "back" end documentation.

This algorithm takes input files of total, diffuse and direct SW irradiance 1-minute measurements, detects clear-sky periods, and fits a curve to the clear-sky measurements using the cosine of the solar zenith angle as the independent variable. It then computes the effect of clouds on the downwelling SW irradiance by subtracting the fitted clear-sky irradiance from the measured irradiance. The clear sky detection algorithm is designed for use not only in climates that experience semi-frequent clear sky conditions, but also those characterized by ubiquitous cloudiness. Clear fit coefficients in regions characterized by semi-frequent clear sky conditions are determined for each day it is possible (see Long and Ackerman, 2000); while ubiquitous cloudy regions fit coefficients are determined using a one-fit-forall approach. Details of each of these approaches will be presented in section 3.3 "Running in Daily Fit Mode” and 3.4 "Running in One-Fit-For-All Mode”. 
The algorithm is designed as an operational data archive-processing tool. The time period of data processed in operational mode is a function of the cloudiness characteristics of the site from which the data was collected. The time period required for a given site is selected to ensure a sufficient amount of "clear-sky" data for fitting. For example, the ARM Program of the U.S. Department of Energy (DOE) has a site in the central U.S. near Lamont, Oklahoma, which represents a "typical” northern mid-latitude plains climate. It is recommended that this algorithm be run on a monthly basis for the Lamont site, since during the summer there can be periods of up to 14 days or more without sufficient clear-sky measurements for daily curve fitting. On the other hand, an Australian site near Leigh Creek has climatologically about 250 to 300 days per year sufficiently clear for daily curve fitting. This site could be processed on a weekly basis. And there are some places, like the tropical western Pacific warm pool region, where clear skies are few and far between. In this case, there are rarely clear periods of sufficient duration to calculate daily coefficients. For sites like this, the algorithm can be set to determine one set of coefficients for the entire data period being processed, this processing period including an amount of historical data as required to ensure enough clear-sky conditions are encountered to provide for a smooth fit.

NOTE: Because Fortran77 does not allow dynamic setting of array size, all array sizes are "hardwired" in to the code. In the present form, the code can handle up to five years worth of 1-minute data files. Should you need to process a longer time series, you can either run several batches, or change the code to handle larger arrays. To do this, look for the following parameter statements in the code, and change them accordingly:

\section{PARAMETER STATEMENTS TO SET ARRAY SIZES:}

PARAMETER (NMAX $=100000)$ - NMAX is the total number of points that can be fit in the arrays for a single fitting.

Used in subroutines: swclreq1, medfit, rofunc, swclreq2, and swclreq3

PARAMETER (NDAYMAX $=18500)$ - NDAYMAX is the total number of days that can be handled in one run of the code.

For 5 years max of data, set NDAYMAX $=1850$

For 50 years max of data, set NDAYMAX $=18500$

Used in subroutines: clrcof1, clrcof2, and swclrfcg

\section{Preparing the Raw Input Files}

The Clear ID code is intended to be able to handle many different forms of input data formats. It uses Fortran77 unformatted read statements to read the input data files, and creates data output files (*.cos) in a format that the rest of the code uses for processing. This portion of the code was made as generic as possible, but there are some "rules" incorporated to make the code practical. The most basic rule, naturally, is that the input file must be compatible with the unformatted read, i.e., values must be comma or space, etc. delimited in each row. Additional rules are as follows:

1) The input data must be in ASCII, chosen as the most basic form of file format. For the ARM SW FLUX ANAL 1Long VAP, the "front end" handles production of the ASCII input files from ARM NetCDF data files. 
2) All data must be in rows, with the date/time listed before the measurement data on each row, then the measurement values. Each row must have the date/time integers (and other integers if you want) first, then the measurement data as reals. After the initial header lines and such (which you set to skip over in the configuration file explained below), all successive lines must contain data until the end of the file. Note that FORTRAN unformatted read declared for REALS does not understand a character "NaN," which is sometimes produced by UNIX-run codes. Thus, if the ASCII input files contain any "NaNs," the code may bomb.

3) The date can be separate integers (i.e., YY or YYYY MM DD, where YY or YYYY is the two digit or four digit year, MM is the two digit month, DD is the two digit day of the month.) Or it can be all one integer, i.e., YYYYMMDD, or YYYYJJJ, where "JJJ" is the day of the year. If the date is separate integers, then they can be in any order. The time info can be separate integers (hh mm, where hh is the hour of the day, and mm is the minute of the hour, no seconds are accepted), or all one integer (hhmm). The code in this case will also accept “day minutes” as one integer, i.e., the number of minutes since midnight. If the time is separate integers, then they can be in any order. Also, no matter whether all one integer or separate, the date and time can be in any order, i.e., the time info can come first. Lastly, the date and time info can be in the form of a single real number of the format YYYYMMDD.hhmmss.

4) The input date and time can either be in Universal Time Coordinates (UTC), or Local Standard Time (LST). Note that LST is not necessarily local (political) time. LST is defined strictly from the site longitude, changing one hour for each 15 degrees of longitude, with the zero change LST time zone (from UTC) centered on Greenwich, England. Thus, the "zero offset” LST time zone covers the longitude from 7.5 degrees East to 7.5 degrees West longitude. The following is the FORTRAN code to determine LST hour offset (hrofst) from UTC:

hrofst $=$ FLOOR((ABS(longit $)+7.500) / 15.000)$

IF(longit.lt.0.0) hrofst $=$-hrofst

where longit is the site longitude ranging from -180 to +180 with minus being west longitude and positive being east longitude, ABS is the absolute value, and FLOOR truncates to the integer portion of the real value (i.e., rounds down). For example, if the site longitude is 116.8 degrees west longitude:

116.8 $\mathrm{W}=-116.8$

$\operatorname{ABS}(-116.8)=116.8$

$(116.8+7.5) / 15.0=(124.3) / 15.0=8.286666666667$

FLOOR(8.286666666667) $=8$

Since longit is negative, thus it is less than zero, Hrofst $=$-hrofst $=-(8)$

Thus, LST in this example is UTC - 8 hrs. 
5) All measurement values to be used by the code must be in "real" numbers. There can be integers in the rows after the final value you will want the code to read, but NO INTEGERS can be "mixed in" in between the last integer at the start of the row and the last input value to be used.

6) The input files must contain values for all four SW components (total, direct, diffuse) even if one of them is just a column of "bad and/or missing data" values such as “-999.0". The code will work if you only have two of the three measurements, but there must be a column for all three VALUES. If you want, you could generate a missing total, direct, or diffuse measurement as the sum/difference of the other two. Unfortunately, if you do not have the total, direct, and diffuse measurements then the code cannot perform the automated data QC, but it will produce fitted clear-sky estimates and cloud effect.

\section{Running the Clear ID and Fitting Algorithm (swclrid1a)}

\subsection{Necessary Files}

This part involves five files: "swclidNz.bat," "swclridNz.cnf," "swclridNz.exe," "swlastdy.cfn,” and "swlastdy.cfw." The process runs by executing the batch file. Note that the batch file contains lines that may be remarked out (so they don't execute) by placing "REM” in front of that line. The important operational parts of the batch file are the last two lines, where a list of files to be processed is piped into a directory file named "inputfils.dir," and the "swclrid1a.exe" executable is called. The DOS command "dir /b /on filelist > outfile" creates a directory listing (dir) of brief file names (/b) in alphabetical order (/on) of filelist (these are your input files) and pipes the list into an output file called "inputfls.dir" (> inputfls.dir). Do not change the name "inputfls.dir"! Note that the line to list the input files includes a directory path. If the input files are in the same directory as the executable, then just put the DOS wildcard-type listing (ex: “19*.ldr”) there. But the code can accept a path character string up to 160 characters in length (listed in the config file below) so that you can run the code in a different directory than where the input files are. The output files, however, are created in the same directory as the executable resides.

Some other lines in the batch (preceding the last two lines) are there primarily for my convenience in developing/debugging the code. Normally you can remark these lines out, or just delete them. The first two lines of the batch file:

copy swlastdy.svn swlastdy.cfn

copy swlastdy.svw swlastdy.cfw

Copy "saved" last day coefficient files to overwrite the operating coefficient files used in initial interpolation. DON'T leave these lines active for operational processing! The two files "swlastdy.cfn" and "swlastdy.cfw" are written and used by the code to keep track of the coefficients of the last "clear enough" fitted day in the previous/current run. The numbers in "swlastdy.cfn” represent, from left to right: date in YYYYMMDD format, diffuse ratio "a" coefficient, diffuse ratio "b" coefficient, total shortwave (TSW) "a" coefficient, and TSW "b" coefficient. These are coefficients for the equation:

$$
\mathrm{Y}=\mathrm{a} *(\operatorname{Cos} \mathrm{Z})^{\mathrm{b}},
$$


where Y is either the clear-sky TSW or clear-sky diffuse ratio (the diffuse ratio is defined as the diffuse shortwave irradiance over the TSW irradiance), CosZ is the cosine of the solar zenith angle, and a and b are the fitted coefficients. Typical mid-latitude values are given: i.e., $0.10,-0.8,1250.0$, and 1.2 respectively. For the "swlastdy.cfw" file, additional values are included, a pair of coefficients for each value being fitted. When run against data characterized by ubiquitous cloudiness and generating a single set of clear-sky fit coefficients for the entire data run rather than daily coefficients, the value of YYYYMMDD found in the swlasdy.cfn file will be zero, but in the "swlasdy.cfw" file YYYYMMDD will reflect the last day for which the one-fit-for-all diffuse ratio coefficient interpolation sub-routine found a usable coefficient (see section 3.4).

The first time ever you run the code on data from a site, you don't really know what the "ball park" coefficients should be for the climate regime and particular set of instruments and their characteristics. So you run the code once, using these "generic" values, which then generates a "real" set of values in these files. Then run the code again on the same data to "really" process the data. After this initial run, the values left in these files ("swlastdy.cfn" and "swlastdy.cfw") are used by the code the next time you run it on, say, the NEXT month's worth of input files. As you can see, this algorithm is intended to be run about once a month, operationally. That's because the code only works up to the last "clear enough" day in the data set. If there are no clear days in the data being processed, the algorithm fails and zeros are written to these two files. That's why it's a good idea at the end of a successful run to copy these two files values to the files "swlastdy.svn" and "swlastdy.svw." That way, after an unsuccessful run you can un-remark these two lines in the batch file and run the code again when you have more data, but still start with the last clear day coefficients.

The other line in the batch file:

copy swclidNz.cnf.siros.e13 swclidNz.cnf,

is to make sure I was consistently using the same configuration file, instead of, say, one for the Lauder, NZ, when processing ARM SGP site E13 files. You can remark this line out.

\subsection{Configuration File (swclidNz.cnf)}

The configuration file for the program is "swclidNz.cnf." Some of the values in it are pretty much set for you. Each line has a preceding explanation line of what values are being set. The format for this file is fixed, so don't change it. (This is another reason I had a backup config file). Some lines you will need to change, others you will not. Here is a "line-by-line" description of the contents of the configuration file, and examples, with a brief explanation following each:

0) Data include 4th var. flag, 0=(none), 1=(SWup for Alb), 2=(other Solar var), 3=(other Non-Solar var)

1

This is an integer value that tells the code what all values you want fitted, in this example set to " 1 " for "SWup for Alb" Meaning as follows: 
- “(none)” sets the code to only produce fits for downwelling total, diffuse, and direct measurements.

- "(SWup for Alb)" sets the code to also produce a fit for surface albedo, using the same "power law" fit equation format as used for the clear total and diffuse ratio. Note that the input data files in this case must contain measurements of upwelling SW. Note also that there isn't any data QC applied to the upwelling SW measurements, though later in the configuration file there are limits set to preclude fitting for snow-covered (i.e., "too bright”) surfaces.

- "(other Solar var)" sets the code to fit some type of solar-driven downwelling ancillary measurement, such as UV, photosynthetically active (PAR), or one spectral channel, etc., using the same "power law" fit equation format as used for the clear total and diffuse ratio. Note that here again no data QC is applied to this input value.

- "(other Non-Solar var)” sets the code to include some data value in the input files, but NOT produce any clear-sky fit coefficients for the value. The values are just copied from the input files and included in the "*.swf” files.

1) The following are integer values for the \# of data for the additional test running mean, the running difrat mean, minimum number/day for daily coef, and whether to calculate daily coefficients $(1=y e s, 0=$ no) ( 4 integers).

\section{1}

The first two integer values tell the code how many minutes of data to use for the running mean and standard deviation calculations used to detect clear skies (21 and 11). The maximum numbers allowed here are both 60 as that is the size of the arrays in the executable. However, normally these numbers are set between 11 and 21 for 1-minute data, as an hour average severely limits the clear detection capability. These should not need to be changed from the current values for 1-minute data, but need to be adjusted as appropriate to give roughly the same time period for lower resolution data (see below). The third integer sets the minimum number of \#-minute data detected as clear in a single diurnal cycle for that day to be used for daily fitting. Or, if the last integer is set to " 0 ", the minimum number of detected clear measurements in a single diurnal cycle needed to include that data in the "one fit for all data” fitting. It is recommended that you don't set this minimum below 90 minutes equivalent (depending on input data resolution) for daily coefficients (Long and Ackerman, 2000). The last integer sets whether to do daily fitting and coefficient interpolation (usual mode), or to only produce one set of fit coefficients for all input data. This last mode is used, for example, in the tropical western Pacific, where the occurrence of extended periods (hours) needed for a daily fit is rare, and thus interpolation between clear periods would involve months. In this situation, it is better to fit to all detected clear-sky periods. When used in this mode, the third integer needs to be reduced from that of "daily" mode, so that brief periods of clear-sky data are included in the fitting (see recommended settings below). However, the value must also be large enough so that the occasional mis-identification of clear sky is not overly included in the fitting. A value of 15 to 30 minutes equivalent minimum number of detected clear-sky \#-minute values is recommended as the limit to set the third integer for "one fit for all” mode. A more detailed explanation of these values can be found in Long and Ackerman (2000). 
In the example setting above for processing 1-minute data, the additional (last iteration) running mean test is set to span 21 minutes (data points), the running diffuse ratio test spans 11 minutes (data points), and it is required that 110 minutes of data (data points) be detected as clear for fitting a given day. For longer averages of input data, about the same time periods should be set, but this means the NUMBER of data points must decrease. Thus, a "rule of thumb" is to take the 1-minute values and divide by the data resolution, rounding up to the nearest integer. Thus, for 3-minute data, the recommended settings would be:

\section{1}

And for 5-minute data:

\section{1}

NOTE that when the code is run in "one fit for all data" mode, then the required minimum number of points per day should be decreased. (Naturally, or you probably wouldn't be running in "one fit" mode to begin with.) For one minute data, the code is usually run requiring at least 20 minutes (data points) be detected as clear in order for them to be included as part of the data pool to be fitted. Thus, for 1-minute data, the settings are:

\section{0}

For 3-minute data:

\section{0}

For 5-minute data:

\section{0}

2) Solar ephemeris info: Lat $(\mathrm{N}=+)$, Long $(\mathrm{E}=+)$, Time Flag (0/1 for LST/GMT).

$$
-45.04169 .680
$$

You need to change these to the appropriate values associated with the input data. These are used to calculate the solar zenith angle values (if not already provided in the input files), and calculate the UTC/LST offset for the output files. The values must be in degrees and fractions of degrees (not in minutes and seconds of lat/long). In this example, the code is configured for 45.04 degrees South latitude, 169.68 East longitude, and the time in the input files is in LST.

3) The following is the total solar normalization exponent ("b" of TSW/cosZ**b), the Normalized Diffuse Ratio Standard Deviation limit, and the delS + Lim factor (*CosZ).

1.180 .00122 .0 
The first real is a "generic" value used in the first pass for detecting clear-sky periods. This should be set at an "average" value of the SW "b" coefficient determined from typical data. After the first iteration, i.e., in all following iterations in the algorithm, the coefficients used to normalize are those determined in the previous iteration. The second real is the limit that the program uses for the standard deviation of the running normalized diffuse ratio in determining if the sky is clear. The limit 0.0012 works well for most systems. However, if you have a particularly "noisy” data logging/ instrument system (for example many MFRSR data sets), you may have to adjust this parameter if you notice that plots of time series of your data that seem to be clear are not being detected as such. A word of warning, though: the program can't distinguish between system noise and a small increase in standard deviation due to either just a few puffy $\mathrm{Cu}$ or slight variations in a thin (perhaps subvisual) cirrus deck for a very noisy system. This limit is set to err on the side of caution, i.e. reject system noise as slightly "cloudy" in order to ensure more accurate clear detection. If you increase this limit, the program isn't as capable of detecting the above cloud scenarios. The third real is the "change with time" envelope limit constant, the value of 2.0 is correct for 1-minute data. This limit is automatically adjusted for lower temporal resolution data, i.e. multiplied by the number of minutes resolution of the data These three values are pretty much okay as they are, and shouldn't need to be changed. A more detailed explanation of these values can be found in Long and Ackerman (2000).

4) The following is for inclusion of solar zenith angle calculation or $Z$ type:

( 0 = yes, calculate; other means data includes: $1=$ Cos $Z, 2=Z$ degrees, $3=\mathrm{Z}$ radians).

\section{0}

This integer value tells the code whether to calculate the solar zenith angle, or whether some form of solar zenith angle information is already included in the input files. Acceptable forms of input are the cosine of the solar zenith angle (CosZ), the solar zenith angle in degrees, or the solar zenith angle in radians. Note that the solar elevation angle is not acceptable input. In this example, the code is set to calculate the solar zenith angle using the lat/long given in setting 2), and date and time info in the input files.

5) The following are the read variables - number of: header lines to skip, integers in each data line, and reals in each data line. Note: only int, then real allowed.

\section{0}

This tells the code some general things about the input file format. The first integer tells the code how many lines to skip at the beginning of each input file (to skip header lines and blank lines). Note that after these lines are skipped, there must be data on each successive line until the end of the file. The second integer tells the code how many integers are in each line of data at the START of EACH LINE, BEFORE the reals containing the measurement data. The third integer tells the code how many reals follow the integers. Note that this last integer can be less than the actual number of reals on each line, as long as the number listed includes all input data the code will be looking for, as set in other parts of the configuration file $(0,2,12,13,14,23,24)$. In this example, the code is set for input files with no header or blank lines (the data starts immediately on the first line), there are six integers at the start of each line, and the code should be prepared to read 10 reals after the initial integers on 
each line. [NOTE: FORTRAN77 can read an integer as a real, but cannot read a real as an integer. So integers and reals can be mixed if all are to be read in as reals, but not if they are to be read in as integers.]

6) Is the date YYYYMMDD, separate integers, YYYYMMDD.hhmmss, YYYYJJJ? (0/1/2/3) 1

Tells the code the format of the date. The acceptable formats are integers: as 4-digit year then 2-digit month, then 2-digit day, or 4-digit year then 3-digit day-of-year, or separate integers. The date and time may also be one real number, with the date followed after the decimal point by the time as hours, minutes, and seconds. In this example, the date information is in separate integers.

7) Of the number of >INTEGERS < declared in 5), which is either date or yr, mn, dy; or which REAL for YYYYMMDD.hhmmss?

\section{3}

This setting tells the code which integers at the beginning of each line contain the date info. In this example, we set setting 6) to tell the code that the date was in separate integers, so we must have three integers here. In the example input files, the year is listed first, followed by the month and day. If we set 6) to "0", “2”, or " 3 ”, we'd only have one integer on this line.

8) Is the year included in data a four or two digit year? $(0=4$ digit $/ 1=2$ digit $)$ ?

\section{0}

This setting tells the code whether the year listed in the input files is 2-digit, or full 4-digit, in the case of separate integers. In this example, the input year is 4-digit.

9) What are the first two digits of the 4-digit year?

This setting is included for the case where the input file lines include only the 2-digit year. The full 4-digit year is necessary for accurate solar ephemeris calculations (if needed), and for the output file naming.

10) Time: 3 integers: (hhmm)/separate int/daymin/YYYYMMDD.hhmmss (0/1/2/3), \# minutes in avg, stamped beginning/mid/end (1/0/-1)

\section{1}

This setting tells the code the format and characteristics of the time information. The code can handle input resolutions of from 1- through 5-minute input data. [Should the input be other than 1-minute, remember that the settings in 1) need to be adjusted appropriately from those values for 1-minute 
data.] In this example, it is set to read separate "hh mm" integers, consists of 1 minute averages, and is stamped at the beginning of the minute. The indication "daymin" is the time in the format of the number of minutes that have elapsed since midnight on the date listed in 7), with a range of 0 to 1339 .

11) Of the number of $>$ INTEGERS $<$ declared in 5), what position is either time or hr and min? (0 for YYYYMMDD.hhmmss)

\section{5}

This setting works in conjunction with setting 10), telling the code which of the integers at the beginning of each line is the time information. In this example, since 10) is set for separate integers, we need to have two integers here. These tell the code that the hour is the fourth integer at the beginning of each line, and the minutes are the fifth integer.

12) Are the direct measurements direct normal (i.e., NIP) measurements? ( $0=y e s / 1=$ no)

\section{0}

This setting tells the code what type of direct SW measurements are included in the input files. The allowed type is either flat plate (sun's rays striking a detector that is aligned parallel to the surface, as with the total and diffuse measurements), or normal incidence (sun's rays striking a detector that is aligned perpendicular to the rays, i.e., measured by a tracking instrument). In this example, the input files contain normal incidence measurements.

13) If solar zenith info is included in the data, which of the $>$ REALS $<$ declared in 5 ) is it (0 for none)?

0

This setting tells the code whether to perform solar ephemeris calculations for each data time, and if not then which of the reals in the input files contain the solar zenith angle information. In this example, the solar zenith angle information is already included in the input files. Note that this setting allows for processing of data from moving platforms such as ships. While the code itself can only calculate solar zenith angles for a stationary site at the lat/long coordinates listed in 2), if the solar zenith angle information is included in the input files, this restraint is removed.

14) Of the $>$ REALS $<$ declared in 5), what position are the TSW, Dif, dir/dirN, and SWu/SolX/Other?

\section{7}

This setting tells the code the location of the reals the code will be processing. They are, in order, the locations of the total (global), the diffuse, the direct, and the ancillary (if chosen in setting 0). In this example, the total is the eighth real, the diffuse the ninth, and the direct normal (from setting 12) is tenth. The upwelling SW (from setting 0) is the seventh real. 
15) What is the low limit for Total SW Irradiance? (real)

1.0

This setting is the lower limit on total SW that the algorithm will include in calculations. Another limit built into the code is a CosZ of 0.0. Fitting within the code requires taking the LOG of the various values, and fitting power law equations with CosZ as the independent variable. Thus, only positive values greater than zero of the total SW, diffuse ratio, albedo, etc., are allowed. Therefore, this value must be set greater than zero (NOT equal to zero). In this example, it is set to 1.0, and all data lines with a total SW less than this value will be excluded from calculations.

16) What is the max limit for clear-sky diffuse SW Irradiance, min CosZ Limit for NDR testing, and max allowable CNDR proportion? (reals),

$\begin{array}{lll}150.0 & 0.3 & 0.7\end{array}$

The first number is the setting for the constant of the diffuse limit test equation (maxdif). Then at a given time, the maximum allowable clear-sky diffuse (diflim) is set to diflim=maxdif*CosZ^0.5 (see Long and Ackerman, 2000). Typically, a value of around 150.0 to 170.0 is reasonable for the diffuse SW Irradiance found in the mid-latitudes. During the winter months, for higher latitudes, or for "hazy" climates such as the tropical western Pacific, it may need to be increased to maybe 200.0 (due to low solar noon sun elevation in the first two cases) if the algorithm seems to be eliminating obviously clear data from fitting near solar noon. An explanation of the SW Irradiance limit is in Long and Ackerman (2000).

The second and third numbers are used in the "one-fit-for-all" mode. The first is a limit in terms of CosZ for testing the diffuse ratio (DR) that is normalized using the one-fit diffuse ratio "b" coefficient (NDR), such that NDR $=\mathrm{DR} / \operatorname{CosZ}^{\wedge} \mathrm{b}$. Only NDR values for CosZ greater than this limit are tested for reasonable clear-sky daily values (see section 3.4 for details of this test). The third number is a multiplicative factor for the clear-sky maximum allowable NDR limit (also explained in section 3.4). An example of values for the TWP site running in "one-fit-for-all” mode:

$\begin{array}{lll}170.0 & 0.30 .7\end{array}$

Where

170.0 is the allowable clear-sky diffuse limit (maxdif) used for diflim=maxdif*CosZ^0.5

0.3 is the CosZ limit for normalized diffuse ratio testing in the "one-fit-for-all” mode, and 0.7 is the multiplier for the maximum allowable NDR limit in the "one-fit-for-all" mode.

17) What is the max ABS difference (tsw/csw - ssw/cssw) for dif \& dir error chk? (real) 0.08

This setting is the limit for the more sophisticated data QC comparison (discussed later in section 4). Since the clear-sky fits produced by this method also include the instrument response characteristics, comparing the ratios of measured to clear-sky for the total pyranometer and the sum (direct + diffuse) 
gives a more sensitive test for inconsistencies than straight irradiance comparisons. Using the ratio, it is possible to more precisely detect when one of the three instruments has a problem.

18) Path for input files up to 160 characters, include final "`”.

C:|rad_datlasciidat।

This is the path for where to get the input files. NOTE that this path MUST BE THE SAME as the path listed in the "swclidNz.bat" batch file that generates the "inputfils.dir" files list file. If no path is listed in the batch file (i.e., the input files are in the same directory as the executable), leave this line blank. NOTE also that if NOT left blank, you MUST include the closing "'”.

19) Following is the number of iterations between initial and final detections (integer).

4

This setting tells the code how many times to iterate the detection - fitting - detection process. This is in addition to the initial and final iterations. The current value of 4 should not have to be changed. An explanation of this iterative process is in Long and Ackerman (2000).

20) Following is the print flag for the *.tst output files $(0 / 1=n / y)$ and night time data $(0 / 1=n / y)$

\section{0}

The first integer is a flag to print out additional files used in development for debugging the code. It should be left set at "0", i.e. do not print out the debug files. The second flag tells the code whether to include only daylight data (0) or 24-hour data in the output files. This last is handy, say, if the input data for LW radiation is carried through (see setting 23). In the above example, we tell the code not to print out the *.tst files, and NOT to include 24-hour data in the output files.

21) Flag for the measurement physical limits testing $(0=$ no, $1=$ use tests $)$ 1

This flag tells the code whether to perform the data QC tests or not. Should you choose not to use the built-in automated data QC, set this value to " 0 ”.

22) The following are the ALBa and ALBb equation max limits for snow problems (both REALs).

\section{$0.4-0.1$}

This setting puts limits on the acceptable albedo fitting "a" and "b" coefficients. These are used to, for example, eliminate periods of snow cover from albedo fitting. Any fitting technique presupposes that the independent variable is the dominant factor in the changes of the variable being fitted for. The rapid change in ground cover albedo characteristics during snow accumulation and melting preclude this assumption, and can produce highly irregular results. Thus, if fitting for the "typical" 
surface characteristics, the first value should be set a bit above the normally expected value of the site surface. The second value sets a limit on the "shape" of the fitted output. Typically, clear-sky albedo increases with increasing solar zenith angle, as the suns rays hit the surface at increasing glancing incidence. Thus, the clear-sky "b" coefficient here should be negative, similar to the diffuse ratio "b" coefficient. This setting puts an upper (max) limit on acceptable value. You will need to study your own data and surface characteristics if you will be fitting albedo to properly set these limits. The values above are for typical US mid-western plains grass surfaces.

23) You may have up to 20 more $>$ REAL $<$ variables printed out in the output files that are in the input files. Enter the number of extra variables you wish to add below, or " 0 ” for none.

2

24) Of the $>$ REALS $<$ declared in 5), which are the ones to be printed out and up to a five character header to be included in the output file (ex: 6 SWup). Note: One set (integer and header) per line.

\section{LWdn}

1 LWup

Settings 23 and 24 allow for the user to include up to twenty additional data (real) values in the *.swf output files, with a five character header value for each. These are included for convenience of the user.

\subsection{Running in Daily Fit Mode}

In climates characterized by semi-frequent clear sky conditions the clear-sky fit coefficients are calculated for each "clear enough" day as defined by Long and Ackerman (2000), and linearly interpolated for cloudy periods. The algorithm stores the coefficients from the last day in the data run that had enough clear measurements to be fitted, thus files from time periods immediately following the data run can be processed and coefficients interpolated between this "last day" and the first day of the new run that has sufficient clear-sky measurements for fitting. A "clear enough day" is defined as a day for which at least the minimum number of points as set in setting 1 points were found to be clear, and these points encompassed a sufficient range of that days possible solar zenith angle. Further details are available in Long and Ackerman (2000).

\subsection{Running in One-Fit-For-All Mode}

To produce a continuous estimate of clear-sky short-wave irradiance in climates that infrequently have clear-sky conditions a single set of clear-sky coefficients are calculated for the entire run rather than a series of daily coefficients. For the total downwelling SW, the one-fit estimates, if adjusted for EarthSun distance, do almost as well as the daily fit mode. However, the diffuse and direct component clearsky estimates do not fare so well using only one set of coefficients. This is because skies may be determined as "clear", but may have differences in haze or aerosol amounts. In these cases, the haze acts to repartition the energy from the direct component, but still scatter the vast majority of the energy in the forward direction, thus including it in the diffuse portion. For all intents and purposes, the total remains the same. The one-fit-for-all mode, after the final "normal" iteration, goes back through the data a final 
time looking for minimum occurrences of the normalized diffuse ratio (NDR) for CosZ greater than the limit set in setting \#16 of the configuration file. The normalized diffuse ratio is calculated as:

$\mathrm{NDR}=(\mathrm{Dif} / \mathrm{TSW}) / \operatorname{CosZ} \wedge(\mathrm{DFRb}+0.04)$,

Where "DFRb" is from the final "one fit" iteration. The code keeps track of the 4 minimum NDR values each day, if the following conditions are met:

For each individual NDR:

1) If the measured diffuse is LESS THAN OR EQUAL TO the diffuse limit (same as in clear sky detection, diflim=maxdif* $\operatorname{CosZ} \wedge 0.5$ )

2) If the NDR is GREATER THAN OR EQUAL TO 0.05

3) If the NDR is LESS THAN OR EQUAL TO a limit calculated as (diflim*mfac/csw)/(cosz**drb)

Where "diflim" is the diffuse limit, "csw" is the calculated clear-sky SW, "drb" is "(DFRb+0.04)", "DFRb" from the final iteration, and "mfac" is a factor less than 1 set in setting \#16 of the configuration file (typically 0.7 for Manus and Nauru)

Then:

4) IF there are 4 data that pass the above tests in a given day:

And:

5) If the standard deviation of the 4 min NDR values is LESS THAN 0.01

If all the above conditions are met, then that days DFRa is replaced with the new value. Then after each day in the run has been examined as above, the coefficients are interpolated between days that did not get new coefficients. For the last day in the run that DID get new coefficients, that date and DFRa coefficient are written into the "Swlastdy.cfw" file, so that the next run of the code can start the DFRa interpolation from there.

At the TWP Manus and Nauru sites data is processed in monthly increments to allow for seasonal changes in weather such as the spring/summer, wet/dry transitions to ensure there are enough clear sky days. Analysis of the data showed that it was necessary to include eight months of historical data in each monthly run to ensure enough clear sky days are encountered to prevent abrupt changes in the fit coefficients. Additional historical data was found to provide little improvement to the smoothness of the fit. Output files are produced only for the month being processed from the last good day in the previous month's run through the last good day in the current run, where "good day" is identified as a day that met the conditions described above and received new coefficients.

\section{Data Qc Tests and Qc Flags}

As alluded to so far, the algorithm includes some automated data quality checks. These tests are intended to ensure that the algorithm does not use "bad" data in the clear-sky fitting. The algorithm has 
been developed as it has to be applied to historical data sets, as well as currently collected data. As such, there is a basic assumption used in these tests and flags, the choice of which was determined by experience with older SW component measurement data sets. That assumption is that, if there is disagreement between the total SW as measured by an unshaded pyranometer, and that derived as the sum of the direct and diffuse SW components, it is more likely that the sum is in error. The reason for this assumption is that the sum is derived using two separate detection instruments (a NIP and shaded pyranometer) mounted on a sun tracking system. The unshaded pyranometer consists of only that, a pyranometer, and nothing else. Thus, the "sum" system is by far the more complex, and generally more susceptible to mechanical, electronic, and computational (solar ephemeris) breakdown, and has double the chance of anomalous obstruction of the detector, etc. This was especially true in the past given the more primitive designs of tracking equipment, compared to more modern tracker systems. The major drawback to unshaded pyranometer measurements is the increased cosine response error, compared to the BSRN recommended sum method. However, as shown in Long and Ackerman (2000), if one takes the ratio of measured over clear fit SW to investigate the effect of clouds on the downwelling SW, by far most of the cosine response error is removed.

In order to decrease the amount of missing or "bad" data, the algorithm tries to "fill in" missing/bad data. For the measurements themselves, first the total SW is checked to make sure it falls within the physically possible limits of $-20 \mathrm{Wm}^{-2}$ and $1500 \mathrm{Wm}^{-2}$. The lower limit includes the typical known IR loss (sometimes referred to as night time) offsets of thermopile pyranometers. The upper limit includes sufficient range so as not to eliminate periods of extreme positive cloud forcing sometimes seen on 1-minute time scales.

The diffuse and direct measurements are checked, to make sure they are not less than $-20 \mathrm{Wm}^{-2}$. No upper limit is checked for these individually, since that is included in the TSW - sum comparison. If either the direct or diffuse fail this test, but the other passes, then the missing or "bad" value is estimated by subtracting the remaining "good" value from the total SW value. The sum is then compared to the total SW. If the absolute difference between the sum and total SW is more than $100 \mathrm{Wm}^{-2}$, then the diffuse value is set to "bad,” i.e., “-999.0.” Naturally, this last test is nullified if either the direct or diffuse has been estimated by differencing the other two components. However, the more sophisticated tests in the final iteration cover this contingency.

In the event that both the direct and diffuse are "bad," then no clear-sky detection is possible for those time periods. However, the algorithm keeps track of the sum - total SW difference for any detected clearsky data. These clear-sky sum - total SW difference data are used to fit (and interpolate) coefficients for a "difference function" similar to the other clear-sky data fitting. In the final iteration, any "missing" sum SW data is "filled in" by using the total SW measurement, and the "difference function" determined and interpolated for that day. These "filled in" sum data are then subjected to the same advanced testing as the original "good" data. The daily coefficients for the "difference function" are included in the "swclreq*.asc" and "clrcoef3.asc" files, and in the header of the "*.swf” output files; along with all the other fit coefficients.

In the final iteration, the code has determined clear-sky estimates for both the total and sum SW. These fits include the instrument characteristics (Long and Ackerman 2000), and thus taking the ratio of the measured over clear fit values (the "cloud effect ratio") greatly removes these characteristics. This affords us another means to more closely check the data for disagreements. We do this by comparing the 
cloud effect ratios for the unshaded pyranometer (TSW) and the sum (SSW). A limit is set in the configuration file for an acceptable difference limit. If the comparison exceeds this limit, the sum, diffuse, and direct data are set to "bad." This test detects when one of the two tracker instruments had a problem, and when the "filling in" of the sum SW using the "difference function" has failed to adequately produce an acceptable approximation of the sum. In addition, the TSW cloud effect ratio is compared to the measured diffuse ratio (diffuse/total SW). If the cloud effect ratio is large (indicating only small cloud effect), but the diffuse ratio is also large (indicating little or no direct component) for a period of time, this indicates that the tracker is out of alignment, i.e. the shaded and unshaded pyranometer measurements are about equal under conditions when they shouldn't be. In this case again the sum, diffuse, and direct data are set to "bad."

The results of these tests are displayed as flags in the *.swf output files, along with a clear detection flag. The flags values, and their meanings, are as follows:

$$
\begin{aligned}
& \text { sflg “sum flag” - for Sum data and other QC } \\
& \begin{aligned}
0 & =\text { OK } \\
1 & =\text { estimated from TSW plus correction function (orig. bad) } \\
-1 & =\text { bad diffuse or direct, and couldn’t estimate } \\
-2 & =\text { CosZ }>0.25, \mid \text { TSW/CSW }- \text { SSW/CSSW } \mid>\text { swrlim } \\
-3 & =\text { CosZ }>0.09, \mid \text { TSW/CSW }- \text { SSW/CSSW } \mid>2 * \text { Swrlim } \\
-4 & =\text { CosZ }>0.12, \text { TSW/CSW }>0.9 \text { AND Dif/TSW }>0.9
\end{aligned}
\end{aligned}
$$

The "-2" and "-3" tests detect when one of either the direct or diffuse is "bad data." The "-4" tests detect when the whole tracker was off, i.e., the Sum is about equal to TSW because the Diffuse is unshaded.

$$
\begin{aligned}
& \text { tflg “total SW flag” - for TSW } \\
& \begin{array}{l}
0=\text { OK } \\
1=\text { TSW }<-20.0 \mathrm{Wm}-2 \text { (bad data) } \\
2=\text { TSW }>1500 \mathrm{Wm}-2 \text { (bad data) }
\end{array}
\end{aligned}
$$

dflg “diffuse flag” - for diffuse

$$
\begin{aligned}
& 0=\text { OK } \\
& 1=\text { Dif }<-20.0 \mathrm{Wm}-2 \text { (bad data) }
\end{aligned}
$$

$2=$ absolute difference between TSW and Sum $>100.0 \mathrm{Wm}-2$ (bad)

$9=$ diffuse estimated from TSW-Dir

rflg “direct flag” - for direct irradiance

$$
\begin{aligned}
& 0=\text { OK (measured) } \\
& 1=\text { original data bad, estimated by TSW-Dif }
\end{aligned}
$$

$-9=$ bad or missing, can't be estimated

clrf clear detection flag

$0=$ not detected as clear-sky

$1=$ this measurement detected as clear-sky and used for fitting 


\section{Contents of the *.SWF Output Files}

The "*.swf" files are the primary algorithm output files, containing all the data that went in and passed the QC tests, QC flags to tell you what data failed which test and if the "measured" value was estimated, and all the clear-sky irradiance estimates. What values are in what columns is explained below, and in the file "swfheadr.txt."

\subsection{Coefficient Data}

Each "*.swf” output file contains the clear-sky fit coefficients retrieved or interpolated for that day. These coefficients are named as follows in the output files:

$\begin{array}{lll}\text { ASCII Files } & \text { ARM NetCDF Files } & \text { Meaning } \\ \text { Date } & \text { coef_date } & \text { date in YYYYMMDD format, based on LST } \\ \text { DFRa } & \text { coef_clearsky_difratio_a } & \text { "a" coefficient calculated for diffuse ratio } \\ \text { DFRb } & \text { coef_clearsky_difratio_b } & \text { "b" coefficient calculated for diffuse ratio } \\ \text { CSWa } & \text { coef_clearsky_gsw_a } & \text { "a" coefficient calculated for clear-sky global SW } \\ \text { CSWb } & \text { coef_clearsky_gsw_b } & \text { "b" coefficient calculated for clear-sky global SW } \\ \text { CSSWa } & \text { coef_clearsky_ssw_a } & \text { "a" coefficient calculated for clear-sky sum SW } \\ \text { CSSWb } & \text { coef_clearsky_ssw_b } & \text { "b" coefficient calculated for clear-sky sum SW } \\ \text { SCORc } & \text { coef_clearsky_dsw_a } & \text { offset coefficient calculated for clear-sky global-sum difference } \\ \text { SCORa } & \text { coef_clearsky_dsw_b } & \text { "a" coefficient calculated for clear-sky global-sum difference } \\ \text { SCORb } & \text { coef_clearsky_dsw_c } & \text { "b" coefficient calculated for clear-sky global-sum difference } \\ \text { ALBa/SolXa } & \text { coef_clearsky_albedo/uvb_a } & \text { "a" coefficient calculated for clear-sky albedo/UV } \\ \text { ALBb/SolXb } & \text { coef_clearsky_albedo/uvb_b } & \text { "b" coefficient calculated for clear-sky albedo/UV } \\ \text { AvgAU } & \text { sun_earth_distance_dailyaverage daily average earth-sun distance in AUs } \\ \text { NOTE: If "Date" is all zeros, then the code was run in "one fit for all data" mode. In this case, to produce the } & \text { daily clear-sky CSW and CSSW, divide the "a" coefficient by the square of the "AvgAU." }\end{array}$

\subsection{Irradiance Data}

The “*.swf” output files also include the following values:

$\begin{array}{ll}\text { ASCII Files } & \text { ARM NetCDF Files } \\ \text { Zdate } & \text { base_time } \\ \text { Ztim } & \text { time_offset } \\ \text { Ldate } & \text { base_time_LST } \\ \text { Ltim } & \text { time_offset_LST } \\ \text { CosZ } & \text { solar_cos_z } \\ \text { AU } & \text { sun_earth_distance } \\ \text { Tsw } & \text { gswfluxdn_measured } \\ \text { Csw } & \text { gswfluxdn_clearskyfit } \\ \text { tswfcg } & \text { gswfluxdn_cloudeffect }\end{array}$

\author{
Meaning \\ date in YYYYMMDD format, based on GMT \\ time in hhmm format, based on GMT \\ date in YYYYMMDD format, based on LST \\ time in hhmm format, based on LST \\ Cosine of the solar zenith angle (used for fitting) \\ Earth - Sun distance in AUs \\ measured downwelling SW from global pyranometer $\left(\mathrm{W} / \mathrm{m}^{\wedge} 2\right)$ \\ clear-sky downwelling SW from global pyranometer $\left(\mathrm{W} / \mathrm{m}^{\wedge} 2\right)$ \\ csw minus tsw $\left(\mathrm{W} / \mathrm{m}^{\wedge} 2\right)$
}




\begin{tabular}{|c|c|c|}
\hline dif & difswfluxdn_measured & measured downwelling diffuse $\mathrm{SW}\left(\mathrm{W} / \mathrm{m}^{\wedge} 2\right)$ \\
\hline Cdif & difswfluxdn_clearskyfit & clear-sky downwelling diffuse SW (W/m^2) \\
\hline diffcg & difswfluxdn_cloudeffect & cdif minus dif $\left(\mathrm{W} / \mathrm{m}^{\wedge} 2\right)$ \\
\hline dir & dirfluxdn_measured & measured downwelling direct SW $\left(\mathrm{W} / \mathrm{m}^{\wedge} 2\right)$ \\
\hline Cdir & dirfluxdn_clearskyfit & clear-sky downwelling direct SW $\left(\mathrm{W} / \mathrm{m}^{\wedge} 2\right)$ \\
\hline difr & difratiodn_measured & measured diffuse ratio (dif/tsw) $\left(\mathrm{W} / \mathrm{m}^{\wedge} 2\right)$ \\
\hline cdifr & difratiodn_clearskyfit & clear-sky diffyse ratio (cdif/csw) $\left(\mathrm{W} / \mathrm{m}^{\wedge} 2\right)$ \\
\hline Ssw & sswfluxdn_measured & measured downwelling sum (dif+dir) SW $\left(\mathrm{W} / \mathrm{m}^{\wedge} 2\right)$ \\
\hline cssw & sswfluxdn_clearskyfit & clear-sky downwelling sum (cdif+cdir) SW (W/m^2) \\
\hline sswfcg & sswfluxdn_cloudeffect & cssw minus ssw \\
\hline Sflg & qc_sswfluxdn & "sum flag" for Sum data and other QC \\
\hline tflg & qc_gswfluxdn & "total SW flag" for TSW QC \\
\hline Dflg & qc_difswfluxdn & "diffuse flag" for diffuse SW QC \\
\hline rflg & qc_dirswfluxdn & "direct flag" for direct SW QC \\
\hline clrf & flag_clearsky_detection & clear detection flag \\
\hline alb/SolX & albedosw/guvbdn_measured & measured albedo (no units) or SolVar (units as in input data) \\
\hline Calb/CSolX & albedosw/guvbdn_clearskyfit & clear-sky albedo (no units) or SolVar (units as in input data) \\
\hline
\end{tabular}

\section{Other Output Files}

After the processing is completed (successfully), there will be many files besides the *.swf files in the directory where the executable was run. Some of these files have uses in such applications as long-term data quality assessment, and a ready record of the "clearest" days in the run. At present, there are no equivalent ARM NetCDF versions of these files; however that may change in the future given experience and user feedback. Brief descriptions:

“*.cos” - files of daylight input data that are generated in the format that all but the front of the algorithm uses for processing. These include all the "good" data (not in the bad_data.cos file) used by the algorithm. These are also the files that contain the "extra" variables set in the configuration file, settings 23 and 24 .

“*.cl2” - files used in the LAST iteration of coefficient fitting. These include all data detected as clear by the algorithm tests, plus all "effectively clear" data. These "effectively clear" data are those that are very close to the next to last iteration coefficient based clear irradiance values, and exhibiting very little, but slightly more than the set limit of, allowable variability, and pass a few other statistical tests that have been determined to eliminate data that do not well represent clear-sky equivalent values (i.e. "magic"). The idea here is to "fill in and extend" the "clear" data for better fitting. The initial clear ID is set to err on the side of caution, sometimes eliminating data that really is clear but a little noisy. Also, the clear ID process tends to eliminate early morning/late evening data due to normalization. This last "trick" allows us to use some of the early morning/late evening data and that data that, if not exactly COMPLETELY clear, are virtually identical to the clear value. This enhances the accuracy of the fitting for large solar zenith angles and/or local solar noon. 
“*.clr” - files containing the "actually detected as clear" data. This same information is now included in the "*.swf" files as a clear flag $(1=$ identified as clear, $0=$ not identified as clear). However, these files might also be useful, since they contain only the clear data for each day.

NOTE: The following files are "generic" file names, and are overwritten each time the code is run. If, for example, you want to keep a record of "bad data," then you MUST rename the bad_data.cos and bad_data.swf files before you run the code again.

“bad_data.cos” - file includes all data that didn’t pass the initial irradiance comparison physical limits tests and were either replaced by differencing if possible, or if not then eliminated entirely from the processing. This file is useful to detect periods when the input data were inconsistent, as described in "swfheadr.txt."

"bad_data.swf” - file includes all data that didn’t pass the algorithm advanced QC tests and were either replaced by differencing if possible, or if not then eliminated entirely from the processing. This file is useful to detect periods when the data were more subtly inconsistent, as described in "swfheadr.txt."

“*.dir" - These files are the various directory file lists that were included in the run. These are helpful in debugging when things go awry, basically to see if the "front end" might have had problems, or whether the problem was internal to the "swclidNz" code.

"cnf_read.asc" - Another debugging tool, this file lists what the code read from the configuration file. This is handy to see if perhaps the configuration file had some lines missing, or not in the proper format, etc.

"swclreq*.asc" - These are the actual clear-sky fit coefficient files produced by the code run. When produced using the daily curve fit, the files are useful for determining when the "clear enough" days occurred in the run, and to give an idea as to how clear each day was as they contain the number of clear data points detected each day. This accounting is handy if one is looking for particular types (clear or cloudy) of days in the data record. These files also contain the local solar noon zenith angle information for each day, and fitting check flag codes from the coefficient tests if the coefficients failed a test(s). (These flags replace the number of detected clear data values.) The test failure codes are as follows:

Flag Action

-44 Not sufficient "high sun” clear points this day. Keep Diffuse ratio "a” and "b” coefficients for this day. Don’t keep the TSW “a” and "b” coefficients, instead interpolate from the last "good” TSW coefficients.

-55 or -99 Not sufficient "low sun" clear points this day, or diffuse ratio "b" coefficient less than 0.95. Keep Diffuse ratio "a” coefficient and the TSW "a” and "b” coefficients for this day. Don’t keep the diffuse ratio "b" coefficient, instead interpolate from the last "good" "b" coefficient. 

this day. Don't keep the diffuse ratio "a” and "b" coefficients, instead interpolate from the last "good" diffuse ratio coefficients.

The file numbers 1, 2, and 3 represent the initial iteration, last "middle" iteration, and final iteration results, respectively. Files numbers 1 and 2 include fitted coefficients for the total SW and diffuse ratio only, file number 3 includes all fit coefficients.

In persistently cloudy locations where a one-fit-for all data approach is needed, the file consists of a single entry with a date of 000000 and the clear-sky fit coefficients, number of clear data points detected, and other information represent the entire data period rather than a single day.

"clrcoef.asc" and "clrcoef3.asc" - These files contain the interpolated clear-sky fit coefficients from the "swclreq*.asc" input for the run. Either daily coefficients are presented or a single set for the entire period depending on the frequency of clear sky periods at the site. If a single set of coefficients are presented they are marked with a date of 0 . "clrcoef.asc" represents the last "middle" iteration values, and includes only the total SW and diffuse ratio coefficients. "clrcoef3.asc" represents the final iteration values, and includes all fit coefficients.

"swclrid*.day" - These files contain information useful for both debugging problems, and as a record of some aspects of the data run. Files 1 and 2 represent the initial iteration and last "middle" iteration, respectively. They contain the number of detected clear-sky data $(\mathrm{N})$, and the number of daylight data points (TotN) for each day. They also include the average normalized diffuse ratio (AvNDR) and average normalized total SW (AvNSW) values for the detected clear-sky data for that day. The "swclrid3.day" file is for the final iteration, and additionally includes the number of data that were considered for detecting clear-sky (N), the number of clear detected via "normal" means (\#clr) and the number detected using the additional *.cl2 method described above (\#cl2). Additionally, the number of "partly cloudy” (\#pcldy), and “overcast” (\#ovc) using a VERY primitive classification system (i.e., only relatively accurate) are included. Comparing these values is useful in describing the type of day each day was. Finally, the local solar noon zenith angle (sncz) and average earth-sun distance in AUs (AvgAU), and the min (czmin)and max (czmax) cosine of the solar zenith angle of the detected clear-sky data, are included. In all, these files are a wealth of descriptive information about the individual days, and how the code treated these days, useful for both characterizing the data set and for debugging.

"posfcg.asc" - This file contains data, in the same format as in the "*.swf" files, for "positive cloud forcing” periods, i.e. when the measured downwelling total SW was significantly greater than the corresponding clear-sky amount. This positive cloud forcing is caused by the enhancement of the downwelling diffuse SW by the presence of clouds, while at the same time the direct SW is not blocked by clouds. This file can be used to study the frequency at which these events occur, and at what magnitude, for each site under study.

"swfcgday.asc" - This file is a daylight average of the data included in the "*.swf” files. The values in this file do not necessarily well represent each days average amount, unless there was little or no 
missing or "bad" data that day. To determine the representativeness, included in these files are the total number of daylight data in the input files (dayN), and the total number of "good" data for each of the total SW (tswn), the total clear-sky SW (cswn), the diffuse SW (difn) and the clear-sky diffuse SW (cdifn) for each day. By looking at the time series of "dayN", you can see what days had significant missing data. Note that the dayN exhibits seasonal changes related to day length, as it changes in respect to solar declination angle changes. Comparing the time series of tswn, cswn, difn, and cdifn (along with DayN) then gives information relating to possible instrument measurement problems in the data set. This information then relates to how well it was possible for the algorithm to perform accurate clear-sky detection and fitting for the data run. The algorithm performance is naturally dependent on the quality and continuity of the data input into the code, i.e. GIGO...

"swnulclr.bat" - kind of left over from earlier versions of the code. This is a batch file that is generated each time, and can be run to delete files that were associated with days that were not clear enough (*.clr) for fitting, or beyond the last “clear enough” day.

"clrndr_b.asc" - for each period processed, this file documents the input *.cos file, the original diffuse ratio a coefficient (DFRa) value, the new DFRa coefficient if the conditions described in section 3.4 "Running in one-fit-for-all Mode" were met, and a flag indicating whether or not the conditions were met. If the conditions were not met the new DFRa value is assigned a value of 9.0000 and the flag is set to 0 , other wise the flag is assigned a value of 1 .

"clrndr_a.asc" - for each period processed, this file documents the date, the one-fit diffuse ratio "b" coefficient (DRb), the NDR test result diffuse ratio a coefficients (OrigDRa) from the file “clrndr_b.asc", and the interpolated DFRa coefficient for that date used in the final estimation.

\section{Running the Fractional Sky Cover Algorithm}

The sky cover algorithm processes the "*.swf" files as input, and is comprised of 3 files in the PC version: "armswzcf.bat," "armswzcf.cnf," and "armswzcf.exe.” As before, the batch file first produces a directory file, then calls the executable. The configuration file sets certain parameters used by the executable. Again, you can set a path (this time up to 600 characters in length) to get the input files elsewhere, output files are generated in the same directory as the executable. The batch file creates a list of the "*.swf” input files.

For this retrieval code, all the configuration file values are set for you and should not need to be changed. The only line you'll need to change is the first one, where the input file path is set. An explanation of the ideas behind this algorithm and the values set for the cloud fraction regression formula can be found in (Long et al. 1999). Again, the format of this configuration file is set for reading by the executable, and must not be changed. The value-by-value description of the configuration file is as follows:

out $\quad / *$ path for input files (incl. trailing “’”) */

This is where the input file path is set.

$17 \quad / *$ number of data (reals) in input files (17 normal plus xtra) */ 
This tells the algorithm the number of reals to expect on each line of the input files. For the "*.swf" files with no extra variables, this value is 17 . This value must be 17 or greater.

$0 \quad / *$ print flag for output files $(0 / 1 / 2 / 3=$ neither/c15/swc/both files) $* /$

This flag tells the code what output files to generate. The code by default produces one file with all the 15-minute averages of the data from the run. The *.swc files are daily files at the same temporal resolution as the input data. The *.c15 files are daily 15-minute average files.

$0 \quad / *$ print flag for fitting output files $(0=$ no files $) * /$

This flag tells the algorithm whether to print out files used for debugging and algorithm development. Leave this set to zero.

$2.255 / *$ Cloud Fraction Equation a coefficient (real) */

$0.9381 / *$ Cloud Fraction Equation b (exponential) coefficient (real) */

These are the empirically derived coefficients for the sky cover estimation equation relating the normalized diffuse cloud effect to sky cover (Long et al. 1999). These were originally left configurable early in the development of the method. However, subsequent study has yet to produce an example of these coefficients not being appropriate. Studies are still in progress using this algorithm, so perhaps a climactic condition wherein these coefficients may have to be adjusted will be found. For now, these should be left set as they are.

$0.37 / *$ Difcld limit for ovc detection */

$0.90 / *$ Diffuse ratio limit for ovc detection */

3 /* number of measurements for running DR StDev */

$0.05 / *$ DR StDev limit for Ovc detection */

As noted in Long et al. (1999), the normalized diffuse cloud effect ratio is not unique as the sky cover approaches unity. This is because thick overcast and heavy cumulonimbus clouds produce depressed, rather than enhanced, diffuse cloud effect. However, the periods of thick overcast and heavy cumulonimbus occurrence also exhibit distinct characteristics of magnitude and temporal variability of the diffuse ratio, total SW cloud effect, etc. These characteristics are detected in the data time series, and the sky cover is set to 1.0 for these times. These configuration settings are part of the overcast detection, and again have always worked as intended for all data sets processed so far. They should be left as they are.

$0.2 \quad / *$ CosZ limit for cloud fraction retrieval */

Because of the increased uncertainty of the SW measurements, the increased percentage uncertainty in the estimated diffuse clear-sky SW, and the sun angle limitations of the original sky imager retrievals used as "truth" in deriving the sky cover equation coefficients, the sky cover estimates by this method increase in uncertainty at low sun angles. This line sets the limit, as the cosine of the solar zenith angle, for which the algorithm will produce sky cover estimates. It is currently set for a CosZ of 0.2 , which translates to a solar elevation angle of about 11 degrees (solar zenith angle of 79 degrees). 
$-8.0 / *$ "bad data" limit for cosz */

$-8.0 \quad / *$ "bad data" limit for au */

-998.0 /* "bad data" limit for tsw */

-998.0 /* "bad data" limit for csw */

Etc...

These lines tell the algorithm what the "bad data" values are for each input value. The algorithm looks for input values LESS THAN the values here, so they must be set slightly greater than the actual "bad data" value. For instance, the "*.swf" "bad data" value for total SW is "-999.0." Thus the limit set here is "-998.0", so that the actual "bad data" value is $1 \mathrm{Wm}-2$ less than this limit.

The executable takes each input file (*.swf) name stem, and generates an output file with this stem and "*.c15" or "*.swc" extension. The c15 output file contains 15-minute averages of all values in the input file (except for the data QC flags), and a 15-minute average fractional sky cover estimate. The value names are the same as those for the "*.swf" files, noted above. Also included in the output files is 4 "flags," listed here as ASCII version name first, followed by the ARM NetCDF version name in parenthesis:

Ncf (nsamples_cloudfraction) - the number of data points that went into this 15-minute average of cloud fraction. Ideally should be 15 .

Ncsw (nsamples_gswfluxdn_clearskyfit) - the number of data points that went into the 15-minute average of CSW (clear-sky fit to TSW). Ideally should be 15. This serves as an indication of the maximum number of data in the original input files that was available during this 15-minutes.

Nssw (nsamples_sswfluxdn_measured) - the number of data points that went into this 15-minute average of SSW (sum of direct + diffuse). Ideally should be 15. This serves as a check of if either diffuse or direct data was missing or bad.

Nclr (nsamples_clearsky_detection) - the number of data points that went into this 15-minute average of cloud fraction that were originally detected as clear by the clear ID algorithm. If Ncf is 15 and this number is 10 , then this means 10 of the 151 -minute periods were detected as clear originally, and the other 5 that went into this average came from the cloud fraction regression formula.

Another output file is generated which is based in part on the date of the first input file name in the directory file, and ending with an ".a15" extension. This file is the same format as the "*.c15” files, but contains ALL the 15-minute data from this run of the code. At present, there is no equivalent ARM NetCDF version of this file.

The "*.swc" output file contains all the data at the same resolution as the input data, including the clear flag for detected clear sky data. In addition, the fractional sky cover (cf) and a flag that tells how the sky cover was determined (cflg) are included. These flags are noted as:

cflg value meaning

ニニニニニニニニニ $=$ ニニニニニ= 


\begin{tabular}{|c|c|}
\hline-9 or -1 & retrieval not possible \\
\hline 0 & detected as clear sky by SWFluxAnal \\
\hline 1 & CosZ less than allowable retrieval limit \\
\hline 2 & $\begin{array}{l}\text { DifCld value negative, meas/clear } \mathrm{SW}<0.4 \text {, } \\
\text { skycov set to OVC }\end{array}$ \\
\hline 3 & $\begin{array}{l}\text { DifCld value negative, meas/clear SW }>0.4 \text {, } \\
\text { skycov set to clear }\end{array}$ \\
\hline $4-6$ & $\begin{array}{l}\text { Overcast detection using DifCld, Diffuse Ratio } \\
\text { and DR Standard Deviation Limits, } \\
\text { skycov set to OVC (4=thick ovc, } 5=\text { med thick ovc, } \\
=\text { normal thickness ovc) }\end{array}$ \\
\hline 7 & sky Cover calculated from diffuse cloud effect \\
\hline
\end{tabular}

Additionally, the average normalized diffuse cloud effect value (DifCld), sample standard deviation of that value (DRStDev), average diffuse ratio (AvgDR), and number of data available (of the number set in 8th line of the config. file) to produce these averages (AvgN) are included.

\section{References}

Long, C. N., and T. P. Ackerman, 2000: "Identification of clear skies from broadband pyranometer measurements and calculation of downwelling shortwave cloud effects.” J. Geophys. Res., 105, No. D12, 1,5609-1,5626. Available URL: http://science.arm.gov/ clong

Long, C. N., T. P. Ackerman, J. J. DeLuisi, and J. Augustine, 1999: "Estimation of fractional sky cover from broadband SW radiometer measurements.” In Proceedings AMS 10th Conf. on Atmos. Rad., June 28-July 2, 1999, Madison, Wisconsin. Available URL: http://science.arm.gov/ clong 